\title{
Facturation de prestations du chapitre 02.03 «psychothérapie déléguée dans le cadre du cabinet médical»
}

Pour pouvoir facturer des prestations du chapitre $02.03 \mathrm{du}$ TARMED «psychothérapie déléguée», il ne faut pas seulement disposer de la valeur intrinsèque requise de tout médecin déléguant, mais également être en possession d'une reconnaissance pour l'unité fonctionnelle correspondante. Il ne s'agit pas d'une tracasserie administrative, mais bien d'une condition posée par les partenaires tarifaires. Ceux-ci exigent en effet que non seulement les médecins qui délèguent la psychothérapie mais aussi les thérapeutes non-médecins qui la pratiquent répondent à des critères de formation précis.
Suite à de longues discussions, la commission paritaire TARMED compétente a donné son feu vert à la FMH pour lancer la procédure de reconnaissance de l'unité fonctionnelle «psychothérapie déléguée dans le cadre du cabinet médical».

Afin de réaliser ce mandat, la FMH a envoyé il y a peu à tous les médecins susceptibles de fournir des prestations du chapitre $02.03 \mathrm{du}$ TARMED une autodéclaration pour l'unité fonctionnelle «psychothérapie déléguée dans le cadre du cabinet médical» accompagnée de la lettre ci-dessous:

Olten, le 20 février 2006

\section{Psychothérapie déléguée dans le cadre du cabinet médical}

Mesdames, Messieurs,

Conformément à la Convention sur la reconnaissance des unités fonctionnelles se rapportant à la convention-cadre TARMED, l'unité fonctionnelle "psychothérapie déléguée dans le cadre du cabinet médical» nécessite une reconnaissance pour pouvoir facturer à la charge des assureurs des prestations figurant dans le chapitre 02.03 du TARMED.

A la demande des partenaires TARMED, la FMH assume la procédure de reconnaissance. Celleci ressemble aux procédures de reconnaissance TARMED pour d'autres domaines et se fonde sur une autodéclaration des fournisseurs de prestations souhaitant fournir et facturer les prestations ad hoc.

Les fournisseurs de prestations entrant en ligne de compte pour la facturation des prestations du chapitre 02.03 du TARMED doivent disposer des valeurs intrinsèques qualitatives suivantes:

- psychiatrie et psychothérapie d'enfants et d'adolescents;

- psychiatrie et psychothérapie;

- attestation de formation complémentaire "psychothérapie déléguée».

Peuvent également facturer ces prestations les médecins qui les ont mentionnées dans le cadre du maintien des droits acquis lors du recensement de la valeur intrinsèque. Ces médecins peuvent dès à présent demander l'attestation de formation complémentaire correspondante. L'adresse de contact est la suivante: Secrétariat de la SSMD/SGDP, Quellenstrasse 31, 8005 Zurich, tél. 04320529 55, e-mail ruthbuchholz@bluewin.ch, Internet www.sgdp.ch.

D'après nos informations, vous faites partie d'un groupe de fournisseurs de prestations susceptible de pratiquer la délégation de la psychothérapie. C'est pourquoi nous vous faisons parvenir en annexe une autodéclaration pour la reconnaissance de l'unité fonctionnelle susmentionnée. Vous y trouverez, au verso, le texte de l'annexe G à la Convention sur la reconnaissance 
des unités fonctionnelles selon le TARMED, laquelle fixe les critères et la procédure de reconnaissance de l'unité fonctionnelle «psychothérapie déléguée dans le cadre du cabinet médical».

$\mathrm{Si}$, dans votre cabinet, vous fournissez des prestations relevant du chapitre $02.03 \mathrm{du}$ TARMED «Psychothérapie déléguée dans le cadre du cabinet médical» et que vous souhaitez continuer à le faire sans rencontrer de problèmes de facturation avec les assureurs, nous vous saurions gré de remplir cette autodéclaration, de la signer et de nous la renvoyer d'ici au 30 mars 2006 à l'adresse suivante: Service tarifaire de la FMH, Gösgerstrasse 8, 4600 Olten.

Etant donné que le nombre de fournisseurs de prestations susceptibles de pratiquer la psychothérapie déléguée est relativement grand, aucun rappel ne sera envoyé. Par conséquent, si nous ne recevons aucune nouvelle de votre part d'ici à la date précitée, nous partirons du principe que votre cabinet médical ne fournit aucune prestation relevant du chapitre 02.03 du TARMED et nous ne vous inscrirons pas sur notre liste.

Veuillez agréer, Mesdames, Messieurs, nos salutations les meilleures.
$\mathrm{FMH}$
Karl Bachofen, responsable du projet
Dr Olivier Kappeler, membre du CC

\section{En résumé}

- Le programme qui règle l'obtention de la formation complémentaire peut être consulté sur le site internet de la FMH à l'adresse suivante: www.fmh.ch/awf $\rightarrow$ formation postgraduée $\rightarrow$ programmes de formation postgraduée et des attestations de formation complémentaire $\rightarrow$ programmes de formation complémentaire $\rightarrow$ psychothérapie déléguée). Est compétent pour l'attribution de l'AFC «Psychothérapie déléguée», le secrétariat de la SSMD, Quellenstrasse 31, 8005 Zurich, tél. 04320529 55, e-mail: ruthbuchholz@bluewin.ch, site internet: www.sgdp.ch.

- L'autodéclaration pour la reconnaissance de l'unité fonctionnelle «psychothérapie délé- guée» peut être consultée sur le site internet de la FMH www.fmh.ch $\rightarrow$ Nos prestations $\rightarrow$ Tarifs $\rightarrow$ TARMED $\rightarrow$ Psychothérapie déléguée; autodéclaration pour cabinet médical. Vous voudrez bien renvoyer l'autodéclaration dûment remplie au Service tarifaire de la FMH, Gösgerstrasse 8, 4600 Olten.

- Pour plus d'informations concernant la reconnaissance de l'unité fonctionnelle «psychothérapie déléguée dans le cadre du cabinet médical», veuillez vous adresser au Service tarifaire de la FMH, Gösgerstrasse 8, 4600 Olten (tél. 06228796 96, e-mail: tarife@fmh.ch). 